\title{
Umgang mit besonderen Herausforderungen bei der ärztlichen Beurteillung von Urteilsfähigkeit
}

\author{
ManuelTrachsela, Daniel Hürlimann ${ }^{\text {b }}$, Helena Hermann ${ }^{a}$, Nikola Biller-Andorno ${ }^{a}$ \\ a Institut für Biomedizinische Ethik und Geschichte der Medizin, Universität Zürich, Schweiz \\ b Rechtswissenschaftliches Institut, Universität Zürich, Schweiz
}

\section{Zusammenfassung}

Urteilsfähigkeit ist eine Grundvoraussetzung für medizinische Behandlungsentscheide wie Zustimmung, Ablehnung oder Abbruch einer Behandlung. Bei PatientInnen mit kognitiven Beeinträchtigungen steht die Urteilsfähigkeit häufig in Zweifel. Neben den viel diskutierten Herausforderungen im Zusammenhang mit der Situationsrelativität und der Kontroverse um mögliche Hilfsmittel zur adäquaten Erfassung der Urteilsfähigkeit stellen kognitive Fluktuationen oft eine besondere Herausforderung für die Beurteilung der Urteilsfähigkeit dar. Dabei handelt es sich um Schwankungen von Kognition, Aufmerksamkeit und Erregung. Im vorliegenden Beitrag werden diese Herausforderungen diskutiert und Vorschläge zur Beurteilung der Urteilsfähigkeit von PatientInnen mit kognitiven Fluktuationen vorgestellt. Abschliessend wird das MehrAugen-Prinzip als Ansatz zur Beurteilung der Urteilsfähigkeit bei besonders folgenschweren Entscheidungen skizziert.

\section{Urteilsfähigkeit und der Stellenwert ihrer ärztlichen Beurteilung}

Urteilsfähigkeit ist eine Grundvoraussetzung für medizinische Behandlungsentscheide wie Zustimmung, Ablehnung oder Abbruch einer Behandlung. Damit handelt es sich um ein Schlüsselelement des verfassungsmässig garantierten Rechts auf Selbstbestimmung von PatientInnen. ${ }^{1}$ Die Beurteilung der Urteilsfähigkeit ist entscheidend für die Verwirklichung zweier ethischer Prinzipien: Dem Respekt vor der Autonomie derjenigen, die fähig sind, für sich selbst zu entscheiden, und die Gewährleistung von Schutz für diejenigen, deren Fähigkeit für selbstbestimmtes Entscheiden eingeschränkt ist. Mit Blick auf diesen ethischen Hintergrund ist eine umsichtige Beurteilung der Urteilsfähigkeit zentral [3-4].

In Anlehnung an die in den USA verwendeten kognitiven Standards zur Beurteilung von Urteilsfähigkeit [5] ${ }^{2}$ hat die Schweizerische Akademie für medizinische Wissenschaften (SAMW) folgende Kriterien vorgeschlagen: (1.) die Fähigkeit, Informationen in Bezug auf die zu fällende Entscheidung zu verstehen, (2.) die Fähig-

1 Das Recht auf Selbstbestimmung ist ein Element des in Art. 10 Abs. 2 BV festgeschriebenen Rechts auf persönliche Freiheit [1-2].

2 (1) Ability to understand the relevant information, (2) ability to appreciate the medical consequences of the situation, (3) ability to reason about the treatment choices, and (4) ability to communicate a choice.

keit, die Situation und die Konsequenzen, die sich aus alternativen Möglichkeiten ergeben, richtig abzuwägen, (3.) die Fähigkeit, die erhaltenen Informationen im Kontext eines kohärenten Wertesystems rational zu gewichten, und (4.) die Fähigkeit, die eigene Wahl zu äussern [6]. Massgebend ist also nicht, wie sich eine Person entscheidet, sondern wie die Person zu ihrem Entscheid kommt (d.h. der Entscheidungsprozess) [7]. Ein Blick in den ärztlichen Alltag in Kliniken und Heimen in der Schweiz zeigt, dass trotz des hohen Stellenwerts einer korrekten Beurteilung der Urteilsfähigkeit diesbezüglich grosse Unsicherheiten bestehen. Eine empirische Untersuchung anhand leitfadengestützter Interviews hat ergeben, dass im Zusammenhang mit fraglicher Urteilsfähigkeit unterschiedliche theoretische Konzepte von Urteilsfähigkeit zur Anwendung kommen [8]. Als herausfordernd beurteilten mehrere Interviewpersonen die Einschätzung der Urteilsfähigkeit, wenn die kognitiven Fähigkeiten der PatientInnen eingeschränkt waren oder eine psychische Störung vorlag. Erwähnt wurden insbesondere Fälle von Demenz, schwer depressiven PatientInnen oder Personen mit psychischen Komorbiditäten [8].

Viele psychische und körperliche Erkrankungen können die Urteilsfähigkeit massgeblich beeinträchtigen [9-11]. Urteilsunfähigkeit ist oft verbunden mit verschiedenen Formen von Demenz, Delirium, Amnesie, Hirnverletzungen, Bewusstseinsstörungen wie Koma, Wachkoma sowie psychiatrischen Erkrankungen wie Schizophrenie und schwere Depressionen oder Bewusstseinsbeeinträchtigungen von kritisch-instabilen Patienten, die aufgrund ihrer Krankheit nicht mehr in die Entscheidungsfindung einbezogen werden können [12]. Die Urteilsfähigkeit ist abhängig von den konkret zu bewältigenden Situationen oder Aufgaben, weshalb von der Relativität der Urteilsfähigkeit gesprochen wird [1316]. So kann eine Person beispielsweise in Bezug auf Alltagsgeschäfte urteilsfähig sein, nicht aber in Bezug auf anspruchsvolle medizinische Entscheidungen. Die Relativität der Urteilsfähigkeit besagt im Grunde, dass eine Person zu einem bestimmten Zeitpunkt im Hinblick auf ein bestimmtes Geschäft urteilsfähig und im Hinblick auf ein anderes nicht urteilsfähig sein kann. Für jede konkrete Situation (bestimmter Zeitpunkt, bestimmte Frage) muss ein dichotomer Entweder-OderEntscheid hinsichtlich der Urteilsfähigkeit gefällt werden. Es gibt keine Abstufungen oder Teil-Urteilsfähigkeit [13, 14, 16]. Für den Fall, dass es im Zusammenhang 
mit einer bestimmten medizinischen Massnahme an der Urteilsfähigkeit fehlt und keine Patientenverfügung vorliegt, ${ }^{3}$ sieht das Zivilgesetzbuch vor, dass bestimmte Personen der Reihe nach berechtigt sind, die urteilsunfähige Person zu vertreten und den vorgesehenen ambulanten oder stationären Massnahmen die Zustimmung zu erteilen oder zu verweigern (Art. 378 ZGB).

Die erwähnte Umfrage [8] hat gezeigt, dass die Mini Mental Status Examination (MMSE) das am häufigsten verwendete Tool ist, das für die Beurteilung von Urteilsfähigkeit herangezogen wird, obwohl dieser Test eigentlich nur als Screeninginstrument für Demenzen und nicht zur Beurteilung der Urteilsfähigkeit geeignet wäre. In den letzten zwanzig Jahren sind verschiedene standardisierte und semi-standardisierte Instrumente zur Erfassung der für Urteilsfähigkeit relevanten mentalen Fähigkeiten entwickelt worden, deren Nützlichkeit jedoch kontrovers diskutiert wird [17-18]. Unter den häufig eingesetzten Assessment-Tools finden sich beispielsweise das Mac Arthur Competence Assessment Tool (Mac-CAT [19]) und das Capacity to Consent to Treatment Instrument [20]. Solche Tools stellen nicht nur eine mögliche Struktur für den Beurteilungsprozess zur Verfügung, sondern helfen der durchführenden Fachperson auch zu dokumentieren, in welchem Ausmass welche mentalen Fähigkeiten betroffen sind und wie sie zu diesem Ergebnis gekommen ist. Je anspruchsvoller eine bestimmte Entscheidung ist, desto ausführlicher und genauer sollte die Dokumentation und Integration in die Krankengeschichte des Patienten erfolgen, unabhängig von den verwendeten Hilfsmitteln.

\section{Kognitive Fluktuationen bei verschiedenen Demenz-Formen ${ }^{4}$}

Kognitive Fluktuationen sind spontane Schwankungen von Kognition, Aufmerksamkeit und Erregung [22] und wurden auch als «Phasen verwirrten Verhaltens, mangelnder Aufmerksamkeit und zusammenhangsloser Äusserungen, unterbrochen durch luzide Episoden mit normaler Aufgabenbewältigung» [23] umschrieben. Auch wenn kognitive Schwankungen ein zentrales Diagnosekriterium der Lewy-Körperchen-Demenz sind, treten diese ebenso bei Parkinson-, Alzheimerund vaskulärer Demenz auf [24-27]. Bei ungefähr 12\% der Patienten mit Alzheimer-Demenz sind kognitive Fluktuationen zu beobachten [23]. Kognitive Fluktuati-

3 Art. 377 Abs. 1 ZGB: «Hat sich eine urteilsunfähige Person zur Behandlung nicht in einer Patientenverfügung geäussert, so plant die behandelnde Ärztin oder der behandelnde Arzt unter Beizug der zur Vertretung bei medizinischen Massnahmen berechtigten Person die erforderliche Behandlung.»

4 Der Text in den Abschnitten «Kognitive Fluktuationen bei verschiedenen Demenz-Formen» und «Kognitive Fluktuationen als Herausforderung für die Beurteilung der Urteilsfähigkeit» basiert auf einem englischsprachigen Artikel von Trachsel, Hermann und BillerAndorno [21]. onen «beeinflussen in signifikanter Weise sowohl die klinische Bewertung der Schwere einer Demenz als auch die neuropsychologische Leistungsfähigkeit» wie z.B. das episodische und semantische Gedächtnis, das Arbeitsgedächtnis, visuell-räumliche Fähigkeiten und das logische Denken [23].

\section{Kognitive Fluktuationen als Herausforderung für die Beurteilung der Urteilsfähigkeit}

Demenz geht einher mit Beeinträchtigungen des Gedächtnisses, der Orientierung, der kognitiven Kontrolle und des Verhaltens [28-30]. PatientInnen mit Alzheimer-Demenz sind im Vergleich zu solchen mit Parkinson-Demenz bezüglich ihrer Urteilsfähigkeit hinsichtlich medizinischer Behandlungsmöglichkeiten stärker beeinträchtigt [31]. Darüber hinaus haben verschiedene Studien gezeigt, dass die Krankheitseinsicht [32] mit dem Fortschreiten der Alzheimer-Demenz abnimmt [33-34]. PatientInnen mit Parkinson-Demenz waren in ihrer Fähigkeit zur Wahl einer Behandlung eingeschränkt. Zudem wurden Korrelationen zwischen exekutiver Dysfunktion als neurokognitivem Faktor und den für Urteilsfähigkeit relevanten mentalen Fähigkeiten bei dieser Patientengruppe gefunden [35].

Ein systematisches Review hat gezeigt, dass durchschnittlich ungefähr $54 \%$ der PatientInnen mit Alzheimer- und $42 \%$ der PatientInnen mit Parkinson-Demenz als urteilsunfähig beurteilt wurden [30]. Für andere Formen der Demenz, darunter auch die Lewy-Körperchen-Demenz, sind derzeit keine solchen Schätzungen bekannt.

Es wurde gezeigt, dass Urteilsunfähigkeit stark von neuropsychologischen Funktionen abhängt wie z.B. Aufmerksamkeit, Konzentration, Gedächtnis, Einsichtsfähigkeit und Argumentationsfähigkeit [36-39]. Defizite in einem dieser neuropsychologischen Bereiche können die Urteilsfähigkeit beeinträchtigen und im schlimmsten Fall zur kompletten Unfähigkeit bezüglich bestimmter Aufgaben oder Entscheide führen [40-41]. Auf Basis dieser Ergebnisse kann davon ausgegangen werden, dass kognitive Fluktuationen häufig mit Fluktuationen der Urteilsfähigkeit einhergehen. Dieser Zusammenhang wurde bisher jedoch nicht empirisch untersucht. Die Urteilsfähigkeit ändert sich nicht nur abhängig von verschiedenen Situationen und Aufgaben (Relativität der Entscheidung), sondern aufgrund kognitiver Schwankungen zusätzlich auch abhängig vom Zeitpunkt ihrer Erfassung [13, 15-16]. Daraus folgt, dass die Untersuchung der Urteilsfähigkeit abhängig von Person, Entscheidung/Aufgabe und Zeitpunkt erfolgen muss [5].

Diese Anforderungen stellen eine schwierige Aufgabe für die beurteilenden ÄrztInnen dar. Die Abhängigkeit von Person, Entscheidung/Aufgabe und Zeitpunkt muss ernst genommen werden, was zur Folge hat, dass die Urteilsfähigkeit sich vom einen Moment zum nächsten 
ändern kann. Gleichfalls verlangt das Gesetz, dass für medizinische Entscheidungen als Voraussetzung für eine eigenständige Entscheidung über medizinische Behandlungen eine klare Einschätzung der Urteilsfähigkeit des Patienten vorliegt. ${ }^{5}$

\section{Vorschläge für die Beurteilung der Urteils- fähigkeit bei Patienten mit kognitiven Fluktuationen}

Die Zuschreibung von Urteilsunfähigkeit betrifft immer nur eine spezifische Aufgabe oder Entscheidung zu einem bestimmten Zeitpunkt. Die beurteilende Ärztin, die eine Urteilsunfähigkeit diagnostiziert, trägt für diese Einschätzung die Beweislast. Bei Kleinkindern oder Personen mit schweren geistigen Beeinträchtigungen ist dieser Nachweis zumindest bei komplexen Entscheidungen unschwer zu erbringen. Wenn Unklarheit über das Vorhandensein oder Nichtvorhandensein der Urteilsfähigkeit besteht, ist solange vom Vorhandensein auszugehen, bis das Gegenteil bewiesen ist (Vermutung der Urteilsfähigkeit und Umkehr der Beweislast) [13-16].

Bei kognitiven Fluktuationen ist es wichtig, dass die Ärztin einen Tag oder Zeitpunkt wählt, an dem sich der Patient in vergleichsweise guter Verfassung befindet. Die Information zu verschiedenen Behandlungsoptionen, Risiken und die informierte Einwilligung (informed consent) sollte während einer solchen Phase erfolgen.

\section{Wie findet man heraus, ob sich PatientInnen aktuell in guter Verfassung befinden?}

Häufig können PatientInnen selbst einschätzen, ob sie sich aktuell in guter Verfassung befinden. Daher sollten sich ÄrztInnen zunächst auf die Meinung ihrer PatientInnen stützen. Daneben besteht auch die Möglichkeit, in Zusammenarbeit mit Angehörigen oder mit dem behandelnden Pflegeteam den idealen Zeitpunkt für die Beurteilung der Urteilsfähigkeit zu finden. Im Normalfall wissen die Angehörigen aus ihrer täglichen Erfahrung im Umgang mit PatientInnen am besten, ob sich die betroffenen Personen gegenwärtig in vergleichsweise guter Verfassung befinden oder ob sie vorübergehend gestresst, niedergeschlagen, aufgewühlt, müde oder in anderweitig reduziertem Zustand sind. Darüber hinaus ist die Hilfe von Verwandten bei der Beurteilung der Urteilsfähigkeit auch aus anderen Gründen wichtig: Die Bedeutung der sozialen Einbettung und intimer Beziehungen für die Entscheidungsfindung von PatientInnen [41] wurde verschiedentlich hervorgehoben [4243]. Persönliche Beziehungen und das soziale Umfeld einer Person beeinflussen deren Autonomie. Weil Verwandte in der Regel am besten wissen, welche Äusse-

5 Eine minimale Relativierung der Dichotomie findet sich allerdings in Art. 377 Abs. 3 ZGB (mit Bezug auf die Erarbeitung eines medizinischen Behandlungsplans): «Soweit möglich wird auch die urteilsunfähige Person in die Entscheidfindung einbezogen.» rungen oder Entscheide ihrer Angehörigen autonom und authentisch sind, ist es wichtig, sie in den Prozess zur Einschätzung der Urteilsfähigkeit einzubeziehen.

\section{Wie kann der Zustand von PatientInnen in schlechter Verfassung verbessert werden?}

In gewissen Fällen kann es notwendig sein, zunächst das zugrundeliegende medizinische Problem, das die kognitiven Fluktuationen verursacht (z.B. Delir), zu behandeln und erst danach die Urteilsfähigkeit einzuschätzen. Dabei ist etwa an eine Änderung der Medikamentendosierungen $\mathrm{zu}$ denken, z.B. eine Reduktion von Benzodiazepinen mit sedierendem Effekt und Verschlechterung der kognitiven Fähigkeiten.

Emotionale Störfaktoren sollten so weit wie möglich reduziert werden, indem äussere Bedingungen geschaffen werden, in denen sich PatientInnen wohlfühlen. Wenn die Beurteilungsbedingungen der Urteilsfähigkeit für eine Person unangenehm sind, können unter Umständen die Anwesenheit und Unterstützung von Verwandten helfen, weil dadurch eine entspanntere, familiärere Atmosphäre geschaffen werden kann.

Zudem sollte die Übermittlung relevanter Informationen in einfacher und verständlicher Sprache erfolgen, wobei auf einen unnötig hohen Detaillierungsgrad zu verzichten ist. Die Vermittlung von Kernaussagen kann zusätzlich erleichtert werden, indem auf Bilder oder Illustrationen zurückgegriffen wird. Auch andere Techniken zur Verbesserung der Urteilsfähigkeit wie zum Beispiel eine Veränderung der Umgebung können hilfreich sein [44-45]. So kann beispielsweise die Verlegung einer Besprechung von der Klinik in die gewohnte Wohnumgebung des Patienten positive Auswirkungen auf dessen Urteilsfähigkeit haben.

Schliesslich ist zu berücksichtigen, dass PatientInnen auch während des Beurteilungsprozesses der Urteilsfähigkeit kurzfristig starken kognitiven Fluktuationen unterliegen können. In diesem Fall sollte ausschliesslich die während den besten kognitiven Momenten gewonnenen Informationen verwendet werden, wobei hier Vorsicht geboten ist, da die Auswahl der besten kognitiven Momente ebenfalls selektiv und vom Beurteiler abhängig ist.

\section{Mehr-Augen-Prinzip bei folgenschweren Entscheidungen}

Die Zuschreibung von Urteilsunfähigkeit hat weitreichende Folgen für PatientInnen, zumal sie dann nicht mehr selber entscheiden dürfen, welche medizinischen Massnahmen sie beanspruchen möchten und welche nicht. Aus diesem Grund schlagen wir das Mehr-AugenPrinzip vor, welches beinhaltet, dass die Zuschreibung von Urteilsunfähigkeit von mindestens zwei ÄrztInnen bestätigt werden muss, wenn es um besonders folgenschwere Entscheidungen geht. Beispiele für folgenschwere Entscheidungen sind der Abbruch einer le- 
benserhaltenden Massnahme oder die Zustimmung zu einem grösseren und besonders riskanten chirurgischen Eingriff. Eine Zweitmeinung muss jeweils nur dann eingeholt werden, wenn die Urteilsfähigkeit aus Sicht der erstbeurteilenden Fachperson fehlt. Auch hier muss konsequenterweise das In-dubio-Prinzip zur Anwendung kommen. Das bedeutet, dass im Falle von fehlender Übereinstimmung zwischen den ÄrztInnen vom Vorhandensein der Urteilsfähigkeit auszugehen ist.

\section{Funding}

The work on this paper is funded within the National Research Program NRP 67 "End of life" of the Swiss National Science Foundation SNSF (SNSF-Grant 406740_139294).

\section{Abstract \\ Handling of particular challenges in the medical assessment of decision-making capacity}

Decision-making capacity is a prerequisite for medical decisions like consent, discontinuation, or refusal of treatments. In patients with cognitive impairments decision-making capacity is often doubted. Besides challenges with regard to situational relativity of decisionmaking capacity and the controversy regarding possible assessment tools discussed elsewhere, cognitive fluctuations are a particular challenge for the evaluation of decision-making capacity. In the present article these challenges are discussed and suggestions for the assessment of decision-making capacity in patients with cognitive fluctuations are made. Furthermore, the multiple-eye principle is outlined as an approach for the assessment of decision-making capacity for particular momentous decisions.

\section{Résumé}

\section{Gestion des situations exceptionnelles lors de} I'évaluation médicale de la capacité de discernement La capacité de discernement est une condition préalable à toute décision concernant un traitement médical, comme le consentement au traitement, le refus ou l'interruption du traitement. Chez les patients présentant des troubles cognitifs, il arrive fréquemment que la capacité de discernement soit mise en doute. En plus des défis soulevés par la relativité de la situation - déjà largement discutés - et de la controverse autour des possibles moyens pour l'évaluation adéquate de la capacité de discernement, les fluctuations cognitives des patients représentent souvent un défi majeur lors de l'évaluation de la capacité de discernement. Il s'agit, à cet égard, de fluctuations de la cognition, de l'attention et des émotions. Ces défis sont discutés dans cet article et des propositions pour l'évaluation de la capacité de discernement des patients présentant des fluctuations de la cognition sont présentées. En conclusion, le principe du contrôle multiple est présenté comme une approche possible pour l'évaluation de la capacité de discernement en cas de décisions lourdes de conséquences.

\section{Korrespondenz}

Dr. med. Dr. phil. Manuel Trachsel

Institut für Biomedizinische Ethik und Geschichte der Medizin

Universität Zürich

Pestalozzistrasse 24

CH-8032 Zürich

E-Mail: manuel.trachsel[at]uzh.ch

\section{Referenzen}

1. Kiener R, Kälin W. Grundrechte, 2. Auflage, Bern: Stämpfli; 2013, S. 147.

2. Schweizer RJ. In Ehrenzeller B, Schindler B, Schweizer RJ, Vallender KA (Hg.). Die schweizerische Bundesverfassung, St. Galler Kommentar, 3. Auflage, Zürich: Schulthess; 2014, Art. 10 N 6.

3. Berghmans R. Capacity and consent. Curr Opin Psychiatr. 2001; 14(5):491-499.

4. Buchanan AE, Brock DW. Deciding for Others: The Ethics of Surrogate Decision Making. Cambridge, England: Cambridge University Press; 1989.

5. Grisso T, Appelbaum PS. Assessing Competence to Consent to Treatment: A Guide for Physicians and Other Health Professionals. Oxford, England: Oxford University Press; 1998.

6. SAMW, Medizin-ethische Richtlinien zur Betreuung von Patientinnen und Patienten am Lebensende, Kommentar ad 2.1. Urteilsfähiger Patient.

7. Berghmans R, Dickenson DR, Ter Meulen M. Mental capacity: in search of alternative perspectives. Health Care Anal. 2004;12(4): 251-263.

8. Graf I, Stettler P, Künzi K, et al. Entscheidungen am Lebensende in der Schweiz: Sozial-empirische Studie nach Konzept und im Auftrag von: Aebi-Müller RE, Dörr B, Gerber AU, Hürlimann D, Kiener R, Rütsche B, Waldenmeyer C, Bern; 2014 (abrufbar unter www.lebensende.ch/studie.pdf).

9. Appelbaum PS, Grisso T, Frank E, O’Donell S, Kupfer DJ. Competence of depressed patients for consent to research. Am J Psychiat. 1999;156(9):1380-1384.

10. Lapid MI. Decisional capacity of severely depressed patients requiring electroconvulsive therapy. J ECT. 2003;19(2):67-72.

11. Palmer BW, Dunn LB, Appelbaum PS, et al. Assessment of capacity to consent to research among older persons with schizophrenia, Alzheimer disease, or diabetes mellitus. Arch Gen Psychiat. 2005;62(7):726-733.

12. Trachsel M, Mitchell C, Biller-Andorno N. Decision-making incapacity at the end of life: conceptual and ethical challenges. Bioethica Forum. 2013;6(1):26-30.

13. Bigler-Eggenberger M, Fankhauser R: In: Honsell H, Vogt NM, Geiser T (Hg.). Basler Kommentar zum Zivilgesetzbuch I, 5. Auflage, Basel: Helbing Lichtenhahn; 2014, Art. 16 N 34 ff.

14. Bucher E. In: Meier-Hayoz A (Hg.). Berner Kommentar zum Zivilgesetzbuch, Kommentar zu den Art.11-26 ZGB, Bern: Stämpfli 1976, Art. 16 N 87 ff.

15. Hausheer H, Aebi-Müller RE. Das Personenrecht des Schweizerischen Zivilgesetzbuches, Bern: Stämpfli; 2012, Rz. $06.50 \mathrm{ff}$.

16. Widmer Blum CL. Urteilsfähigkeit, Vertretung und Selbstbestimmung, Diss. Luzern, Zürich 2010, S. 32 f. und 38

17. Lamont S, Jeon Y, Chiarella M. Assessing patient capacity to consent to treatment: an integrative review of instruments and tools. J Clin Nurs. 2013;22(17-18):2387-2403.

18. Hermann H, Trachsel M, Biller-Andorno N. Accounting for intuition in decision-making capacity: Rethinking the reasoning standard? Philosophy, Psychiatry, Psychology; accepted for publication. 
19. Grisso T, Appelbaum PS. MacArthur Competence Assessment Too for Treatment (MacCAT-T). Sarasota, FL: Professional Resource Press 1998.

20. Marson DC, Ingram KK, Cody HA, Harrell LE. Assessing the com petency of patients with Alzheimer's disease under different legal standards. Archives of Neurology. 1995;52:949-954.

21. Trachsel M, Hermann H, Biller-Andorno N. Cognitive fluctuations as a challenge for the assessment of decision-making capacity in patients with dementia. American Journal of Alzheimer's Disease and Other Dementias; 2014; Epub ahead of print.

22. McKeith IG, Dickson DW, Lowe J. Diagnosis and management of dementia with Lewy bodies: Third report of the DLB Consortium. Neurology. 2005;65(12):1863-1872.

23. Escandon A, Al-Hammadi N, Galvin JE. Effect of cognitive fluctuation on neuropsychological performance in aging and dementia. Neurology. 2010;74(3):210-217.

24. Ballard C, Aarsland D, McKeith I, et al. Fluctuations in attention: PD dementia vs DLB with parkinsonism. Neurology. 2002,59(11): 1714- 1720 .

25. Ballard C, O'Brien J, Gray A, et al. Attention and fluctuating attention in patients with dementia with Lewy bodies and Alzheimer disease. Arch Neurol. 2001;58(6):977-982.

26. Walker MP, Ayre GA, Cummings JL. Quantifying fluctuation in dementia with Lewy bodies, Alzheimer's disease, and vascular dementia. Neurology. 2000;54(8):1616-1625.

27. Metzler-Baddeley C. A review of cognitive impairments in dementia with Lewy bodies relative to Alzheimer's disease and Parkinson' disease with dementia. Cortex. 2007;43(5):583-600.

28. Starkstein S, Sabe L, Petracca G, et al. Neuropsychological and psychiatric differences between Alzheimer's disease and Parkinson's disease with dementia. J Neurol Neurosur Psychiat. 1996;61(4): 381-387.

29. Martin RC, Okonkwo OC, Hill J, et al. Medical decision-making capacity in cognitively impaired Parkinson's disease patients without dementia. Movement Disord. 2008;23(13):1867-1874.

30. Sessums LL, Zembrzuska H, Jackson JL. Does this patient have medical decision-making capacity? JAMA. 2011;306(4):420-427.

31. Warner J, McCarney R, Griffin M, Hill K, Fisher P. Participation in dementia research: rates and correlates of capacity to give informed consent. J Med Ethics. 2011;34(3):167-170.
32. Dawns M. Awareness in dementia: in the eye of the beholder. Aging Ment Health. 2005;9(5):381-383.

33. Zanetti O, Vallotti B, Frisoni GB, et al. Insight in dementia: when does it occur? Evidence for a nonlinear relationship between insight and cognitive status. J Gerontol Psychol Sci. 1999;54(2):100-106.

34. Defanti CA, Tiezzi A, Gasparini M, et al. Ethical questions in the treatment of subjects with dementia. Part I. Respecting autonomy: awareness, competence and behavioural disorders. Neurol Sci. 2007;28(4):216-231.

35. Dymek MP, Atchison P, Harrell L, Marson DC. Competency to consent to medical treatment in cognitively impaired patients with Parkinson's disease. Neurology. 2001;56(1):17-24

36. Moye J, Karel MJ, Gurrera RJ, Azar AR. Neuropsychological predictors of decision-making capacity over 9 months in mild-tomoderate dementia. J Gen Intern Med. 2006;21(1):78-83.

37. Palmer BW, Savla GN. The association of specific neuropsychological deficits with capacity to consent to research or treatment. J Int Neuropsychol Soc. 2007;13(6):1047-1059.

38. Dreer LE, Devivo MJ, Novack TA, Krzywanski S, Marson DC. Cognitive predictors of medical decision-making capacity in traumatic brain injury. Rehabil Psychol. 2008;53(4):486-497.

39. Baumann H. Reconsidering relational autonomy. Personal autonomy for socially embedded and temporally extended selves. Analyse Kritik. 2008;30(2):445-468.

40. Griffith HR, Dymek MP, Atchison P, Harrell L, Marson DC. Medical decision-making in neurodegenerative disease: Mild AD and PD with cognitive impairment. Neurology. 2005;65(3):483-485.

41. Marson DC, Harrell L. Executive dysfunction and loss of capacity to consent to medical treatment in patients with Alzheimer's disease. Semin Clin Neuropsychiatry. 1999;4(1):41-49.

42. Christman J. Relational autonomy, liberal individualism, and the social constitution of selves. Phil Stud. 2004;117(1/2):143-164.

43. Wirshing DA, Wirshing WC, Marder SR, Liberman RP, Mint J. Informed consent: assessment of comprehension. Am J Psychiatry. 1998;155(11):1508-1511.

44. Dunn LB, Jeste DV. Enhancing informed consent for research and treatment. Neuropsychopharmacology. 2001;24(6):595-607.

45. Kim SYH. Evaluation of Capacity to Consent to Treatment and Research. Oxford, England: Oxford University Press; 2010. 